\title{
A System for Visualization of Power Consumption
}

\author{
Keisuke Miura $^{\mathrm{a}}$,Yoshikazu Hitaka ${ }^{\mathrm{b} *}$, Koichi Ishida $^{\mathrm{c}}$ \\ ${ }^{a}$ Advanced Course of Production System Engineering, Ube National College of Technology, Ube \\ 755-8555, Japan \\ ${ }^{\mathrm{b}}$ Department of Electrical Engineering, Ube National College of Technology, Ube 755-8555, Japan \\ ${ }^{\mathrm{c}}$ Department of Mechanical \& Electrical Engineering, Tokuyama College of Technology, Syunan \\ 745-8585, Japan \\ * Corresponding Author: hitaka@ube-k.ac.jp
}

\begin{abstract}
In recent years, power-saving strategies have become very active. For further developments in order to push ahead with power saving, visualization of power consumption is needed. Thus, we propose a real-time home-based unit that measures, power consumption and provides a visualization of the data for energy consumers, which is more suitable than existing commercial products. This system shows the measured results in real-time on PC. Power consumption is measured by a non-contact current sensor and a commercial step-down power. Then, the measured result is indicated after sending it to PC by wireless communication.
\end{abstract}

Keywords: power consumption, non-contact current sensor, visualization.

\section{Introduction}

The present, due to the desire to minimize global warming on a global scale, limits for $\mathrm{CO}_{2}$ emission have been set and various policies have been implemented ${ }^{(1-6)}$. At the Toyako Summit in 2008, goals were set to reduce world greenhouse gas emissions by $50 \%$ by 2050 . At the L'Aquila Summit in 2009, reductions of $80 \%$ were suggested for all advanced countries. The same year, then Prime Minister Hatoyama of Japan at the United Nations Climate Change Summit expressed a $25 \%$ reduction compared to the 1990 level $^{(7)}$. After this decision, power and energy saving was demanded through changes in the behavior of citizens in family homes ${ }^{(8)}$.

As a result of the great East Japan Earthquake on March 11, 2011, a hydrovolcanic explosion and meltdown broke out in the Fukushima first Nuclear Power Plant. As of 2010, the plant had 50 facilities, of which only two function after the accident. Because the nuclear power generation that accounted for $30 \%$ of the total power generation of Japan became virtually zero, electric power shortages became a problem all over the country ${ }^{(9)}$. To cope with this problem, all electric power companies requested for power savings above $10 \%$; power-saving activities were thus implemented all over the country.

However, an attitude survey on power saving after the earthquake suggested that "the effect of power saving is incomprehensible," $"(10)$ that is, it need to visualize of power saving measures. Therefore, it is suggested that a personal unit for the visualization of power consumption is useful.

In addition, there is an average power saving of $11 \%$ which indicates power consumption as feedback ${ }^{(11,12)}$.

Several companies release real-time measurement and visualization systems. However, most commercially available systems cannot measure the power consumptions of appliances, e.g. ceiling lamps and bracket. There are problems related to attaching sensors in circuit breakers and measuring the current. The measurement accuracy of existing systems is good, but cables and exclusive software are necessary to display data on PC. Alternatively, systems that do not require special hardware for displaying data tend to have poor measurement accuracy.

Thus, we propose a real-time measurement and visualization system for power consumption in a personal home-based unit that overcomes some of the problems of the commercially available products. 


\section{System summary}

The system comprises a measurement circuit including a non-contact current sensor, wireless communication module using Bluetooth, and data display tool for the PC. The non-contact current sensor measures the AC current and can simply be clipped onto the power line in the distribution board. The measurement of the $\mathrm{AC}$ voltage was performed using a step-down transformer. A wireless Bluetooth communication module is used for communication between the measurement circuit and PC, allowing the data to be collected and visualized on the PC. This method enables the system to perform real-time measurements and visualization of power consumption (detailed as follows) and as shown in Figs. 1, 2, and 3.

1) A non-contact current sensor the power supply line leading to the circuit breaker in the distribution board and the AC current flowing though the power supply line was measured.

2) This signal and that measured by the depressed AC voltage signal at the step-down transformer were passed through the bias circuit. After that, the signal amplitude after $\mathrm{A} / \mathrm{D}$ conversion was measured.

3) From this measurement result, effective value of $A C$ current, power, and power consumption were derived. This data was sent to PC via Bluetooth.

4) The transmitted data was displayed by a custom application made for this device.

5) This procedure enabled the system to perform the real-time measurements and visualization of power consumption data.

\subsection{Non-contact current sensor}

This system employed a non-contact current sensor for the measurement of the AC current flows. A schematic of sensor is shown in Fig. 4. When the non-contact current sensor is clipped around an electric wire, it measures and displays the AC current whose magnitude is dependent on AC, $\mathrm{I}_{\mathrm{in}}$, flowing through the electric wire. The output AC current, $\mathrm{I}_{\mathrm{out}}$, is expressed by the following equation, where $\mathrm{n}$ is the number of windings. The frequency is $60 \mathrm{~Hz}$, and the non- contact current sensor outputs an $\mathrm{AC}$ current of the sane frequency.

$$
\mathrm{I}_{o u t}=\frac{I_{\text {in }}}{n}
$$

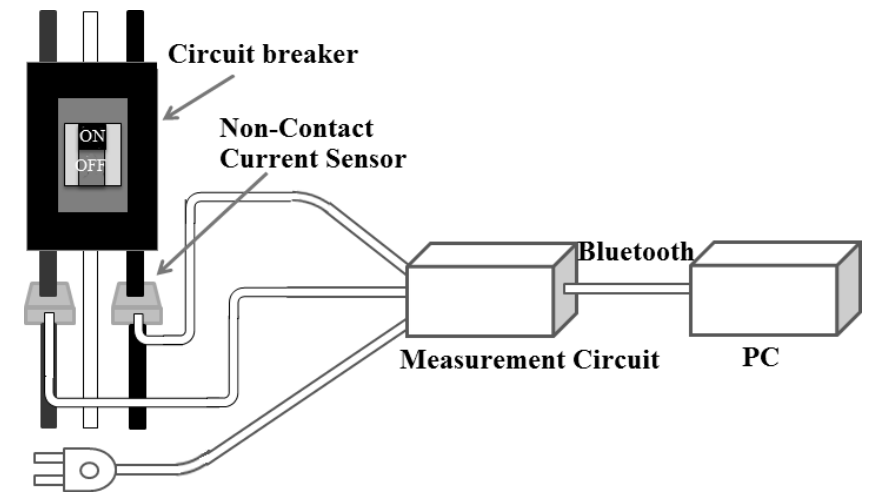

Fig. 1. Overall System

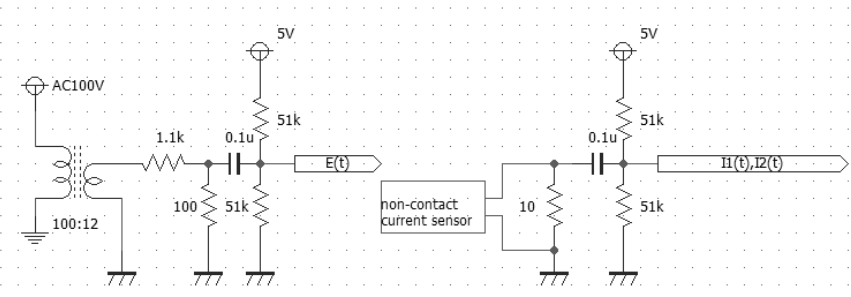

Fig. 2. Measurement circuit diagram (left: AC voltage, right: AC current)

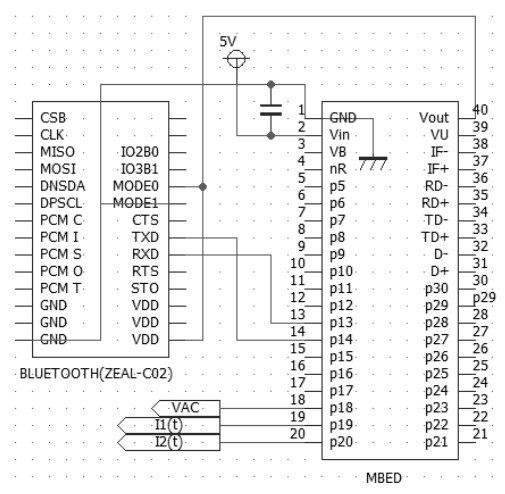

Fig. 3. Measurement circuit diagram(calculator)

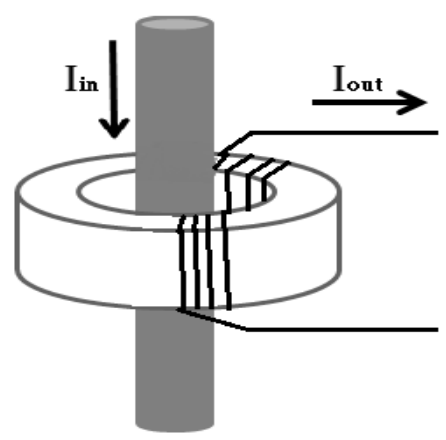

Fig. 4. Schematic of the non-contact current sensor 


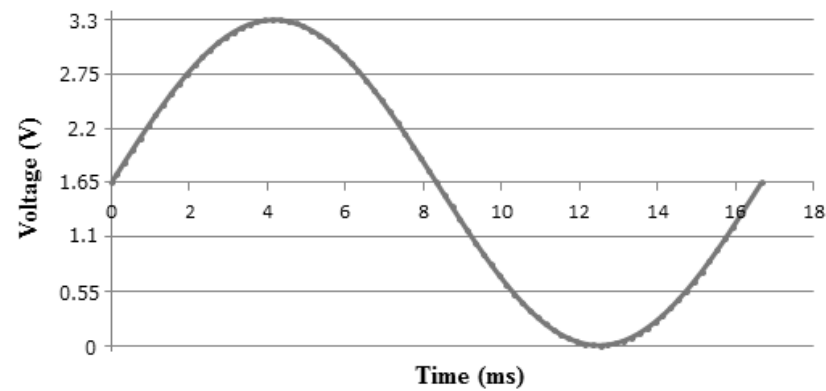

Fig. 5. Waveform chart

\subsection{Measuring circuit}

The measuring circuit is comprised a non-contact current sensor, an amplifier, a transformer, and an mbed microcontroller. The output electric current converted the DC current using a bias circuit after voltage conversion. The bias circuit was adjusted to a median value of $1.65 \mathrm{~V}$ of the rating voltage of the analog input pin of the mbed microcontroller (Fig. 5). The AC voltage was measured using the bias circuit after step-down of the commercial power supply by the transformer. The actual value of the AC current, power, and power consumption were eventually derived by inputting these signals into the mbed microcontroller.

\subsection{Microcontroller (mbed)}

An mbed-LPC1768 was used to calculate power consumption and the down-link to a wireless communication circuit. The effective value of AC current calculates by the $\mathrm{A} / \mathrm{D}$ conversion values of input signals.

When this system turns on a power supply, the signal front the non-contact current sensor and the depressed signal from the transformer are input into the bias circuit. Then, this signal is scaled to a standard $1.65 \mathrm{~V}$ by the bias circuit. The mbed microcontroller reads the instantaneous value of each signal from the A/D converor (sampling period of $73 \mu \mathrm{s}$ ) and calculates the difference between the read and standard value. Next, it multiply the difference from the standard value of current signals and voltage signals. And add the calculation results. Every second this process was interrupted, a calculation of the effective value of the AC current, power, and power consumption was made. After this calculation, the system transmitted data to the PC via Bluetooth.

\subsection{Data visualization application}

The data transmitted by the wireless communication was displayed in an application developed using VisualStudio2010, as shown in Fig. 6.

This application can be distributed; hence, to use this system, it is not necessary to have a copy of this software.

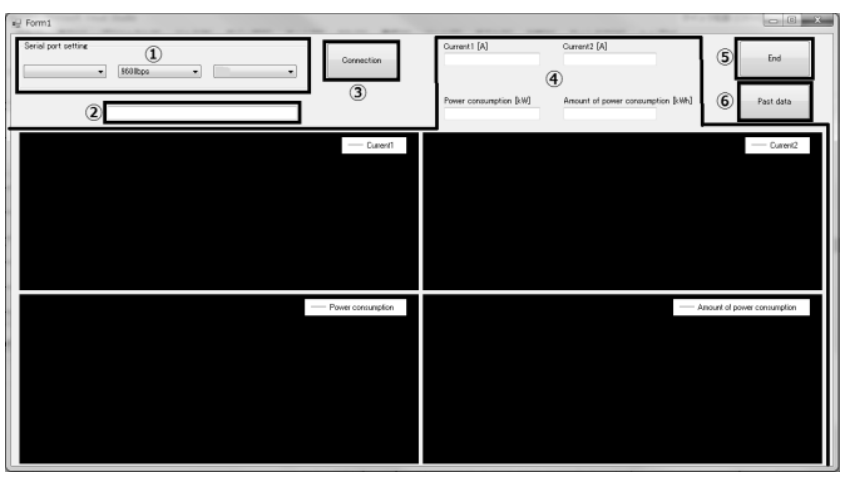

(1) Serial port setting

(2) Current data and hour

(3) Connection/Disconnection button

(4) Measurement result display

(5) End button

(6) Show past data display screen button

(a) Data display screen

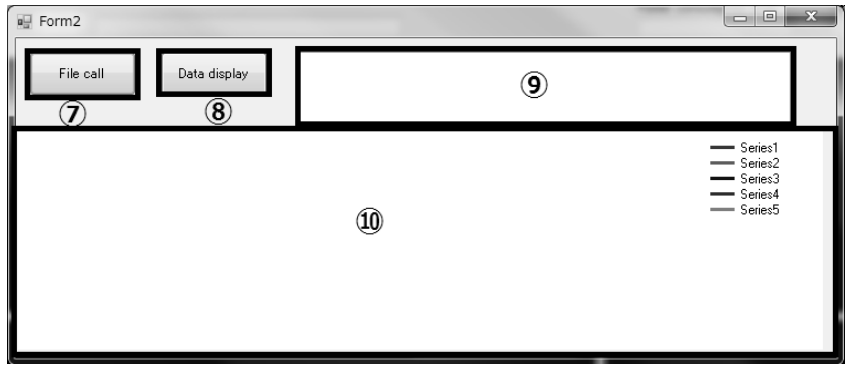

(7) File call button for past data

(8) Past data display button

(9) File name display screen

(10) Past data display screen

(b) Past data display screen

Fig. 6. Application display 


\section{Testing the system}

\subsection{Experimental methodology}

These experiments were conducted using a household switchboard by the following procedures for checking the operation and calculating the measurement accuracy of this system.

1) The power supply plug of the measurement-side system is connected to an outlet.

2) The power supply is turned on find each standard value, which is calculated by the PC at the same time.

Simultaneously, the application on the computer is turned on.

3) The non-contact current sensor is clipped to the power supply line leading to the circuit breaker. At the same time, the value read by the electric meter is recorded.

4) The value from the electric meter is recorded every 30 min. After constant timekeeping, and the power consumption is calculated for each data point and transmitted to the PC.

5) From the measurements of the power consumption and change of the electric meter every $30 \mathrm{~min}$, the measurement accuracy of the system is determined.

6) Using the above-mentioned method, the system operation was verified.

In addition, the formula for calculating the used power consumption and the measurement accuracy of the system is described by the following equation:

$$
W=\frac{1}{3.6 \times 10^{6} T} \sum_{t=0}^{T} E(t)\left\{I_{1}(t)+I_{2}(t)\right\}
$$

where $W$ is the power consumption $(\mathrm{kWh}) ; E(t)$ is the instantaneous voltage of power supply $(\mathrm{V}) ; I_{l}(t), I_{2}(t)$ are the instantaneous currents that flows through each power supply line; $T$ is the A/D conversion time per second.

$$
\delta=\frac{W_{m}-W_{r}}{W_{r}} \times 100
$$

where $\delta$ is error rate $(\%) ; W_{m}$ is the power consumption every $30 \mathrm{~min}(\mathrm{kWh}) ; W_{r}$ is the change of the electric meter value every $30 \mathrm{~min}(\mathrm{kWh})$.

\subsection{Experimental results}

Using the above technique, the determined values of the change of the electric meter, power consumption, and error rate are shown in Table 1 and Figs. 7 and 8, it can be seen that the measurement accuracy is satisfactory, $\pm 10 \%$.

\begin{tabular}{|c|c|c|c|}
\hline $\begin{array}{c}\text { Measurement } \\
\text { times }\end{array}$ & $\begin{array}{c}\text { Electric } \\
\text { meter value } \\
(\mathrm{kWh})\end{array}$ & $\begin{array}{c}\text { Measurement } \\
\text { value } \\
(\mathrm{kWh})\end{array}$ & $\begin{array}{c}\text { Error rate } \\
(\%)\end{array}$ \\
\hline 1 & 0.28 & 0.30 & 8.07 \\
\hline 2 & 0.27 & 0.26 & -4.07 \\
\hline 3 & 0.27 & 0.26 & 2.76 \\
\hline 4 & 0.23 & 0.24 & 6.44 \\
\hline 5 & 0.33 & 0.33 & -1.09 \\
\hline 6 & 0.25 & 0.24 & -2.37 \\
\hline 7 & 0.21 & 0.20 & -3.73 \\
\hline 8 & 0.24 & 0.23 & -3.65 \\
\hline 9 & 0.29 & 0.26 & -9.97 \\
\hline 10 & 0.43 & 0.43 & -0.27 \\
\hline 11 & 0.40 & 0.44 & 8.80 \\
\hline 12 & 0.27 & 0.26 & -5.31 \\
\hline 13 & 0.28 & 0.26 & -5.49 \\
\hline 14 & 0.26 & 0.25 & -5.05 \\
\hline 15 & 0.26 & 0.23 & -10.45 \\
\hline 16 & 0.16 & 0.17 & 7.29 \\
\hline 17 & 0.52 & 0.50 & -3.02 \\
\hline 18 & 0.35 & 0.34 & -2.65 \\
\hline 19 & 0.32 & 0.29 & -8.98 \\
\hline 20 & 0.58 & 0.62 & 6.86 \\
\hline 21 & 0.76 & 0.68 & -9.88 \\
\hline 22 & 0.37 & 0.40 & 9.40 \\
\hline 23 & 0.26 & 0.25 & -3.56 \\
\hline 24 & 0.27 & 0.26 & -3.65 \\
\hline
\end{tabular}

Table1. Measurement results

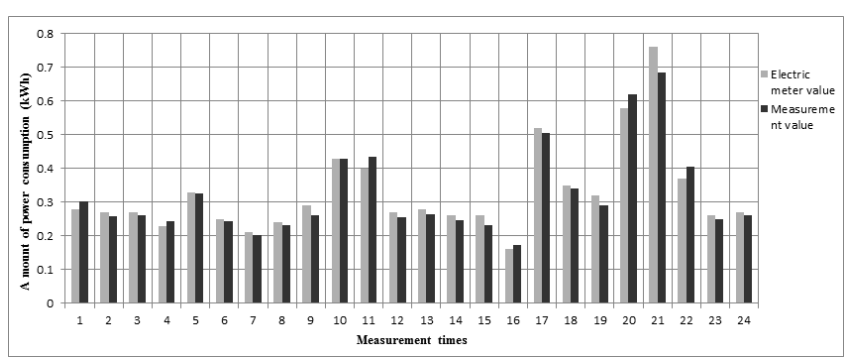

Fig. 7. Power consumption at each measurement time 


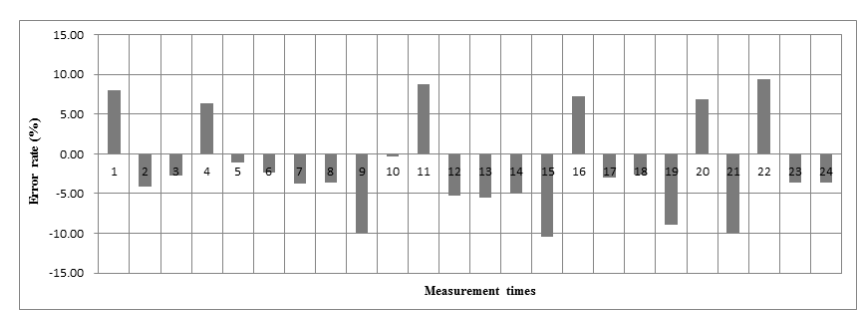

Fig. 8. Error rate at each measurement time

\section{Conclusions}

In this study, we presented a system for real-time measurement and visualization of the power consumption of an entire home using a non-contact current sensor. This system has few limitations as data can be sent to the PC via wireless communication. Furthermore, exclusive software was not necessary for data visualization. In addition, we aimed to improve the measurement accuracy by decreasing the sampling distance.

The measurement accuracy of this system was satisfactory $\pm 10 \%$. A data display screen and the indication screen for the past data functioned smoothly. However, the precision of this system is inferior to that of a commercial product, which has high measurement accuracy. The improvement of the measurement accuracy could be achieved in the future.

\section{References}

(1) Haruo Iguchi, "Recent Public Opinion Polls and Politics in United States Concerning Climate Change(Global Warming)", Journal of Human Environmental Studies, Vol. 5, No. 2, pp. 99-106, 2007

(2) Shintaro Shiina, "Policies and Laws for the Prevention of Global Warming", Proceedings of the Symposium on Global Environment, Vol. 14, pp. 309-317, 2006

(3) Fujio Suda, "Renewable Energy Use and its Policy in the United Kingdom", Journal of Advanced Science, Vol. 13, No. 4, 2001

(4) Hiroyuki Ishitobi, "Current Situation on International Negotiation and Other COP 3 Efforts",Waste Management Research, Vol . 8, No.6, pp. 413-420, 1997

(5) An Wena, Yu Jing, "EU Low-carbon Revolution's Influence on the Yangtze River Delta Regional Development and Its Countermeasures", Energy Procedia, Vol. 5, pp. 2289-2302, 2011

(6) Hiroshi Ohta, "International Cooperation and
Institutional Selection in International Environmental Problems: The Politics of International Policy Alliance over the Issues of Climate Change", TRENDS IN THE SCIENCES, Vol. 16, No. 6, pp. 35-41, 2011

(7) Resources and Energy Agency, "Energy white book 2012"

(8) Hajime Yoshida, "A Consideration on the Policy Development for the Realization of the Low-Carbon Cities-Focusing Largely on the Civilian sector of the Member Cities of the Promotion Council for the Low-Carbon Cities (PCLCC)", Journal of the City Planning Institute of Japan, Vol. 46 No. 3, pp. 1000-1010, 2011

(9) Mitsuyuki Maeda, "A Transformation of Energy Industries in a Less Energy Era", Development Engineering, Vol. 31, No. 1, pp. 41-49, 2011

(10) Public interest incorporated foundation cooperative research institute Foundation, Questionnaire result report about power saving and the energy, 2011

(11) Yukiko Yoshida, "Development of Energy Monitoring System For Residents", AIJ Journal of Technology and Design, Vol. 17, No. 35, pp. 249-253, 2011

(12) Kenji Hiranuma, Masataka Kawana, Masahiro Osakabe, Hiroyasu Kifune, Kunihiko Mouri, Kazutomo Inukai, "Energy Saving Experiment by Smart Meter", Japan Society of Mechanical Engineers Transactions (B reviews), Vol. 79 No. 799, pp. 234-238, 2013 